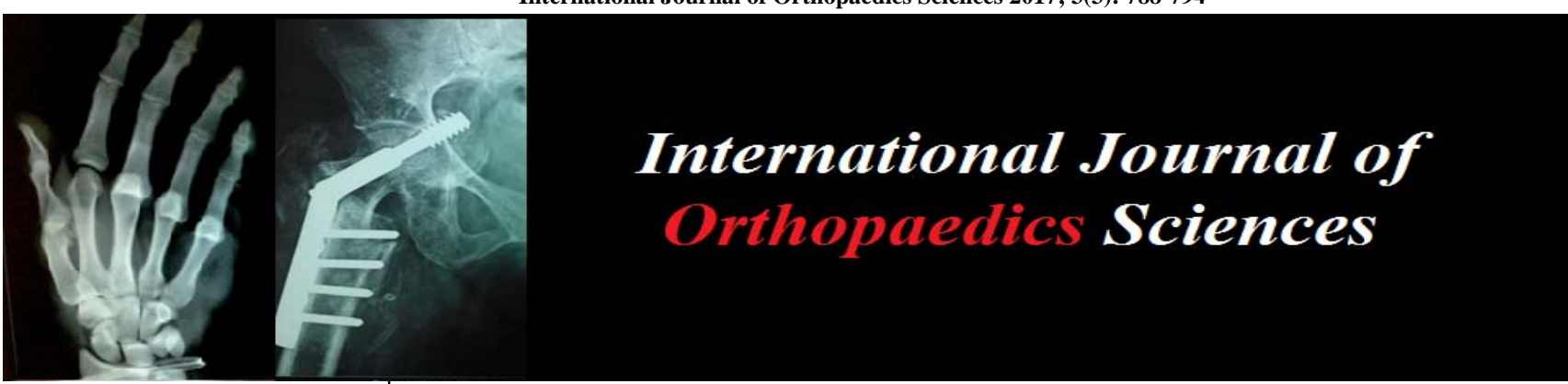

ISSN: $2395-1958$

IJOS 2017; 3(3): 788-794

(C) 2017 IJOS

www.orthopaper.com

Received: 25-05-2017

Accepted: 26-06-2017

J Naveen Chandra

Department of Orthopaedics, Sri

Devaraj Urs Medical College,

Kolar, Karnataka, India

Naga Kumar JS

Assistant Professor, Department of Orthopaedics, Sri Devaraj Urs Medical College, Kolar,

Karnataka, India

\section{PV Manohar}

Professor, Department of

Orthopaedics, Sri Devaraj Urs

Medical College, Kolar,

Karnataka, India

\section{Correspondence}

Naga Kumar JS

Assistant Professor, Department

of Orthopaedics, Sri Devaraj Urs

Medical College, Kolar,

Karnataka, India

\section{A study of surgical management of intertrochanteric fracture of femur with dynamic hip screw}

\author{
J Naveen Chandra, Naga Kumar JS and PV Manohar
}

DOI: $\underline{\text { https://doi.org/10.22271/ortho.2017.v3.i3k.119 }}$

Abstract

Background and Objectives: Intertrochanteric fracture is a leading cause of hospital admissions in elderly people. The number of such admissions is a rise because of increasing life span \& sedentary habits. Conservative methods of treatment result in malunion with shortening and limitation of hip movement as well as complications of prolonged immobilization like bed sores, DVT and respiratory infections. This study is done to analyze the surgical management and traumatic fractures using Dynamic Hip screw.

Materials and Methods: This is a prospective study of 30 cases of fresh intertrochanteric fractures admitted to R.L. Jalappa hospital attached to Sri Devaraj Urs Medical College, Kolar between September 2008 and September 2010. Cases were taken according to inclusion and exclusion criteria i.e., patients with intertrochanteric fracture above the age of 18yrs. Medically unsuitable and old malunited intertrochanteric fractures were excluded from the study. Operation

was done keeping the Tip Apex Distance (TAD) into consideration.

Results: The study shows sex ratio of 7:3 (M:F) with maximum presentation between 51 and 65yrs with an average of 54yrs. Mode of injury was more due to fall, 24(80\%); followed by RTA, 6(20\%). Right side was slightly more than Left side. R:L $=19$ (63.3\%):11 (36.6\%). 13.3\% of patients had associated injury. Type II fractures were maximum with 19 cases followed by Type III\&I $5 \& 4$ cases each. Post operative results were found to be excellent in 6(20\%), Good in 13(43.3\%), Fair in 10(33.3\%) and poor in $1(3.3 \%)$.

Conclusion: This study shows that intertrochanteric fracture is common in elderly population with male preponderance mainly occurring between 51-65 years. Common mode of injury being trivial fall, right side being slightly more involved in injury. Early operative intervention with Dynamic hip screw with $135^{0}$ side plate with barrel gives good results, helps early mobilization of elderly patients decreasing morbidity \& mortality and achieves rigid fixation even in osteoporotic bone.

Keywords: Intertrochanteric fracture femur, Dynamic hip screw, Hip fractures

\section{Introduction}

Intertrochanteric fractures are common in the elderly people. The frequency of these fractures has increased primarily due to the increasing life span and more sedentary lifestyle brought on by urbanization. Intertrochanteric fractures occur in the younger population due to high velocity trauma, whereas in the elderly population it is most often due to blunt trauma. The incidence of intertrochanteric fractures is more in the female population compared to the male due to osteoporosis ${ }^{[1]}$. In a Swedish study of more than 20,000 patients, the incidence of hip fractures in women doubled every 5.6 years after the age of 30 years ${ }^{[2]}$. Inspite of the advances in anesthesia, nursing care and the surgical techniques, hip fractures remain a significant cause of morbidity and mortality in the elderly population. The intertrochanteric fractures can be managed by conservative methods and there is usually union of the fracture. If suitable precautions are not taken the fracture undergoes malunion, leading to varus and external rotation deformity at the fracture site and shortening and limitation of hip movements ${ }^{[3]}$. It is also associated with complications of prolonged immobilization like bedsores, deep vein thrombosis and respiratory infections. Since this fracture is more common in the elderly patients, the aim of treatment should be prevention of malunion, and early mobilization. Taking all the factors into consideration, surgery by internal fixation of the fracture is ideal choice. There are various forms of internal fixation devices used for intertrochanteric fractures 
of them the most commonly used device is the Dynamic Hip Screw with Side Plate assemblies. This is a collapsible fixation device, which permits the proximal fragment to collapse or settle on the fixation device, seeking its own position of stability. In view of these considerations, this study is taken up to analyze the surgical management of Intertrochanteric Fracture using Dynamic Hip Screw and its outcomes regarding the union of the fracture and early mobilization of the patient.

\section{Materials and Methods}

The clinical material for the study of surgical management of Intertrochanteric Fracture of Femur with Dynamic Hip Screws consists of 30 cases of intertrochanteric Fracture of traumatic etiology meeting the inclusion and exclusion criteria, admitted to R.L. Jalappa Hospital and Research Centre attached to Sri Devaraj Urs Medical College from September 2008 TO September 2010.

\subsection{Inclusion Criteria}

- All patients with intertrochanteric fractures of femur

- $\quad$ Age - Patients above the age of 18 years.

- $\quad$ Sex - Both male and female.

\subsection{Exclusion Criteria}

- Patients below the age of 18 years.

- Patients with malunited trochanteric fractures treated elsewhere.

- Medically unstable patients who are an extremely poor anesthetic risk.

- $\quad$ Patients with pathological fractures.

As soon as the patient was admitted, a detailed history was taken and a meticulous examination of the patient was done. The patient's radiographs were taken in the Antero-Posterior and Lateral views; The diagnosis was established by clinical and radiological examination. In the study, the intertrochanteric fractures were classified according to the Boyd and Griffin classification as follows -

Type I: Fractures that extend along the intertrochanteric line from the greater to the lesser trochanter.

Type II: Comminuted fractures, the main fracture being along the intertrochanteric line but with multiple fractures in the cortex. Is a deceptive fracture in which an anteroposterior linear intertrochanteric fracture occurs as in type I, but with an additional fracture in the coronal plane, which can be seen on the lateral roentgenogram.

Type III: Fractures that are basically subtrochanteric with at least one fracture passing across the proximal end of the shaft just distal to or at the lesser trochanter. Varying degrees of comminution are associated.

Type IV: Fracture of the trochanteric region and the proximal shaft, with fracture in at least two planes.

Skin or skeletal traction was applied till the patient was taken up for surgery. Medical evaluation and stabilization of the patient was begun in consultation with the Physician and, the Cardiologist if necessary. Hygiene of the skin was maintained with regular betadine scrub wash. The operative site (lateral aspect of the thigh) was shaved, scrubbed with savlon and betadine scrub, and painted with betadine and spirit, and draped in a sterile towel three days prior to the surgery. The patients were taken up for surgery after obtaining written and informed risk consent of the nature and complications of the surgery. All patients were started on antibiotics prophylactically. Cephalosporins were used. It was administered in the dosage of $1 \mathrm{gm} \mathrm{IV}$, prior to induction of anesthesia, and continued at 12 hourly intervals for 3-5 days, and switched over to oral form till the $14^{\text {th }}$ day postoperatively, i.e. until suture removal.

\section{Operative Technique \\ 3.1 Anaesthesia}

The patients were taken up for surgery under General, Spinal or Epidural Anesthesia.

\subsection{Patient Positioning}

The patients were positioned supine on the fracture table with a radiolucent padded counter traction post placed between the patient's legs. The uninjured leg was held in wide abduction by a boot attached to one of the leg extensions of the fracture table. The injured leg was held in slight abduction, by a boot attached to the other leg extension of the fracture table. The C-arm image intensifier was positioned between the patients' legs and the adequacy of both the antero posterior and true lateral views are verified, before surgical preparation.

\subsection{Reduction Technique}

Closed reduction of fracture by manipulation under image intensifier control was performed.

After the anesthetised patient was positioned on the fracture table, and the extremity was secured in the traction foot piece, traction was exerted longitudinally on the slightly abducted injured leg until reduction was achieved. The degree of rotation required for reduction was variable, depending on the degree of comminution. In non-comminuted fractures without displacement, the limb was fixed in neutral or slight internal rotation. In comminuted fractures, $15^{\circ}$ to $20^{\circ}$ of external rotation was required to close the defect posteromedially. Reduction was checked in the antero-posterior and lateral views, paying special attention to the posterior and medial cortical contact. If reduction was not achieved by closed manipulation, Open Anatomical reduction was done by opening the fracture site. If anatomical reduction was not achieved by either closed or open techniques, a Stable reduction was achieved by opening the fracture site and pegging the medial spike in the proximal fragment into the medullary cavity of the distal fragment. If there was a deficiency in the posteromedial cortex even after stable reduction, cancellous bone grafting was done.

\subsection{Exposure}

The incision of exposure was the standard lateral approach. The incision began $5 \mathrm{cms}$, proximal and anterior to the greater trochanter, curving distally and posteriorly over the posterolateral aspect of the trochanter and then distally along the lateral surface of the thigh, parallel with the femur for about $10 \mathrm{cms}$. The dissection was deepened in the line of the incision down to the fascia lata. The fascia lata was incised with a scalpel in the distal part of the wound and split proximally with the scissors. In the proximal part of the wound, the fascia was divided just posterior to the tensor fascia latae muscle. By retraction of the dissected fascia the vastus lateralis muscle and its origin from the inferior border of the greater trochanter was viewed. The muscle fascia was split laterally; the muscle was dissected from its deep surface posteriorly, and divided near the linea aspera. The body of the 
vastus lateralis was retracted anteriorly, and the perforating arteries were coagulated, if they were divided. After dividing the muscle along the femur, it was elevated with a periosteal elevator and the lateral and anterolateral surface of the femoral shaft was exposed. By further subperiosteal elevation of the proximal part of vastus lateralis and- intermedius, the intertrochanteric line and the anterior surface of the femur, just below this line was exposed. Alternatively, Vastus lateralis was divided at its origin from the greater trochanter transversely, down to the posterolateral surface of femur. Then the muscle and its fascia was divided longitudinally with scissors beginning on the posterolateral surface, $0.5 \mathrm{em}$ from its attachment to the linea aspera.

\subsection{Guide Pin Insertion}

The level of insertion of the pin was approximately $2 \mathrm{cms}$ below the intertrochanteric ridge. An opening was made with a perforation drill in the lateral cortex, midway between the anterior and posterior cortex of the femur. A fixed angle guide was placed on the lateral cortex. The guide pin with $3.2 \mathrm{~mm}$ tip was aimed towards the apex of the femoral head, confirming the central placement of the pin on both anteroposterior and lateral views using C-arm the guide pin was passed with in $10 \mathrm{~mm}$ of the joint. The length of the guide pin inserted was measured, and the guide pin was advanced to an additional $5 \mathrm{~mm}$ into the subchondral bone to avoid guide pin pullout while reaming.

\subsection{Reaming the femur for lag screw and plate barrel}

The cannulated, combination reamer was set to the length of the lag screw measured. The reamer was slided over the guide pin, and femur was reamed coaxial to the guide pin, to avoid bending of the guide pin. Image intensifier was used to know the advancement of the reamer. The reaming was stopped when the short barrel notch indicator on the barrel reamer reaches the lateral cortex.

\subsection{Tapping of femoral head}

The lag screw tap was set for the appropriate lag screw length and inserted into the reamed portion over the guide. Tapping was done under image intensification till the previously reamed portion.

\subsection{Insertion of Lag Screw and Barrel Side Plate}

The appropriate lag screw was assembled onto the insertion wrench. The assembly was placed over the guide pin and introduced into the reamed hole. The lag screw was advanced into the femoral head to the predetermined level and its position was verified with image intensification in both planes. When the screw insertion was complete, the handle of the insertion wrench was perpendicular to the axis of the femoral shaft, which allowed proper keying of the lag screw to the plate barrel. The lag screw retaining rod was unscrewed and the insertion wrench was removed from the back of the lag screw. Then the side plate was advanced onto the lag screw shaft. Later the guide pin was removed.

\subsection{Attachment of the side plate}

The length of the plate was selected depending on the extension of the fracture into the shaft of the femur. The plate was secured to the shaft of femur with a plate clamp. With a $3.2 \mathrm{~mm}$ drill, holes were drilled into the lateral cortex, through the bone screw holes of the side plate. The appropriate cortical screw length was measured with a bone screw length gauge. The holes were tapped with a $4.5 \mathrm{~mm}$ tap. The screws were inserted using a screwdriver.

\subsection{Compression of the fracture}

Inter fragmentary compression was obtained using the barrel compression instrument. The compression screw was threaded into the distal end of the lag screw shaft. The traction on the leg was released and compression screw was tightened to compress the fracture. The position of the lag screw, side plate and fracture compression was confirmed by image intensification in both antero- posterior and lateral views.

\subsection{Closure of the wound}

The vastus lateralis muscle was approximated and sutured with an absorbable suture material. Fascia lata and subcutaneous tissue was sutured with absorbable after placing a suction drain. Skin was sutured with non-absorbable suture material. Sterile dressing was put after removing plastic isolation drape.

\section{Post-Operative Management}

Post- Operative radiographs were taken on the following day. Quadriceps exercises was started on the following day. The patients were encouraged to do active hip and knee movements as soon as the pain and inflammation subsided. Suture removal was done on the $10^{\text {th }}-14^{\text {th }}$ day. Patient was discharged after suture removal. After suture removal active mobilization of the hip and knee was started with nonweight bearing with crutches or a walker until 2 weeks. Partial weight bearing with walking aids was begun from $2^{\text {th }}$ week onwards. Full weight bearing was started after 12 weeks. Follow up X-Rays were taken at 1 month, 3months, 6 months and after 1 year and 2 years. Follow up was done on OPD basis. The follow up was done by clinical and radiological evaluation, and the results were assessed based on Pain, Swelling in the trochanteric region, Deformity, Movements at the hip and knee joints, Limb length, Sitting cross-legged and squatting, Walking, Fracture union and Patient satisfaction.

\section{Results \\ 5.1 Age}

The age of the patients in the study, ranged from twenty three to eighty eight, average being 54.7

Table 1: Showing Age Distribution

\begin{tabular}{|c|c|c|}
\hline Age in yrs & Number of patients & Percentage \\
\hline $20-35$ & 6 & 20 \\
\hline $36-50$ & 7 & 23.3 \\
\hline $51-65$ & 9 & 30 \\
\hline$>65$ & 8 & 26.6 \\
\hline
\end{tabular}

\subsection{Sex}

In the study, out of thirty patients, nine were females and twenty one were males.

Table 2: Showing Sex Distribution

\begin{tabular}{|c|c|c|}
\hline Sex & Number of patients & Percentage \\
\hline Female & 9 & 30 \\
\hline Male & 21 & 70 \\
\hline
\end{tabular}



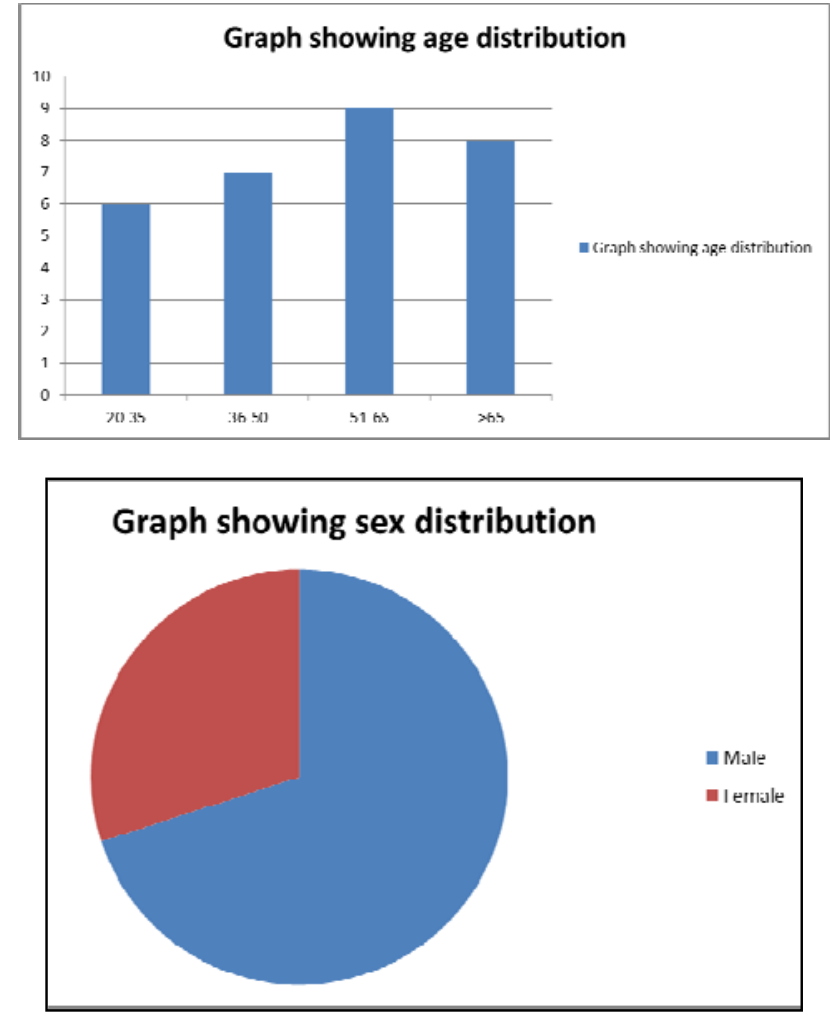

Table 3: Showing Mode of Injury

\begin{tabular}{|c|c|c|}
\hline Mode of injury & Number of patients & Percentage \\
\hline Fall & 24 & 80 \\
\hline Road Traffic Accident & 6 & 20 \\
\hline
\end{tabular}

Table 4: Showing Side Affected

\begin{tabular}{|c|c|c|}
\hline Side affected & Number of patients & Percentage \\
\hline Right & 19 & 63.33 \\
\hline Left & 11 & 36.66 \\
\hline
\end{tabular}

Table 5: Showing Associated injuries

\begin{tabular}{|c|c|c|}
\hline Associated injuries & Number of patients & Percentage \\
\hline Yes & 4 & 13.33 \\
\hline No & 26 & 86.66 \\
\hline \multicolumn{2}{|r}{} \\
\hline
\end{tabular}

Table 6: Showing Type of fracture

\begin{tabular}{|c|c|c|}
\hline Type & Number of patients & Percentage \\
\hline Type I & 4 & 13.33 \\
\hline Type II & 19 & 63.33 \\
\hline Type III & 5 & 16.66 \\
\hline Type IV & 2 & 6.66 \\
\hline
\end{tabular}

Table 7: Showing total duration of hospital stay

\begin{tabular}{|c|c|c|}
\hline Total duration of stay & Number of patients & Percentage \\
\hline$<2$ weeks & 10 & 33.33 \\
\hline $2-3$ weeks & 14 & 46.66 \\
\hline $3-4$ weeks & 4 & 13.33 \\
\hline$>4$ weeks & 2 & 6.66 \\
\hline
\end{tabular}

\subsection{Pain}

In the present study of the thirty patients only three patients had pain in the hip region at the end of six months. It was due to implant cut through in one patient and trochanteric prominence in the other patient. The other patient was an elderly patient who had not complied with the rehabilitative protocol.

\subsection{Swelling}

Only two patients had swelling in the trochanteric region at the end of six months, one due to adduction deformity and prominence of trochanter and the other patient due to prominence of trochanter due to malunion of trochanter.

\subsection{Deformity}

Of the thirty patients only one patient had an adduction deformity due to implant cut through.

\subsection{Range of movements}

At the end of 6 months 4 patients had full ROM, 12 had restriction of terminal flexion and abduction, 8 patients had flexion upto $90^{\circ}$ and abduction of $20^{\circ}, 1$ patient had marked restriction of movements.

Table 8: Showing Movements

\begin{tabular}{|c|c|c|}
\hline Range of movements & $\begin{array}{c}\text { Number of } \\
\text { patients }\end{array}$ & Percentage \\
\hline Full & 9 & 30 \\
\hline $\begin{array}{c}\text { Restriction of all terminal } \\
\text { Movements }\end{array}$ & 12 & 40 \\
\hline $\begin{array}{c}\text { Flexion upto } 90^{0} \text {,Abduction of } \\
20^{0} \text {,internal and external rotation of } 15- \\
20^{0}\end{array}$ & 8 & 26.6 \\
\hline Marked restriction of all movements. & 1 & 3.4 \\
\hline
\end{tabular}

\subsection{Shortening}

At the end of six months out of the thirty patients, 24 patients did not have any shortening, 6 patients had shortening. Shortening was due to fracture collapse as the patient started progressive weight bearing ambulation.

\subsection{Sitting Cross-Legged and Squatting}

At the end of six months, 8 patients were not able to sit crosslegged or squat and the remaining 22 patients were able to sit cross-legged and squat

\subsection{Walking}

At the end of six months, 2 patients were walking with support and the remaining 28 were walking without support.

\subsection{Limp}

At the end of six months, 6 patients had an associated limp while walking and the remaining 24 did not have any associated limp while walking. The limp was because of shortening in the six patients.

\subsection{Complications}

Of the thirty patients in the study, four patients had a superficial wound infection post operatively delaying wound healing, one patient had a cut-out of the Richard's screw from the head and the remaining patients had no complications.

\subsection{Union}

In the present study, in 18 (60\%) cases the fracture had united by twelve weeks and in the remaining $11(40 \%)$ cases the fracture united by the sixteenth week. The average time for radiological union is 16.2 weeks. In the study, none of the cases developed avascular necrosis of the femoral head or secondary osteoarthritis of the hip joint following surgery during the period of follow up. Results were assessed and evaluated using Babhulkar's criteria for evaluation of results of DHS fixations. There were 6 cases with excellent results, 13 cases with good results, 10 cases with fair results and 1 case with poor result. 
Table 9

\begin{tabular}{|c|c|c|}
\hline Result & Number of patients & Percentage \\
\hline Excellent & 6 & 20 \\
\hline Good & 13 & 43.3 \\
\hline Fair & 10 & 33.3 \\
\hline Poor & 1 & 3.3 \\
\hline
\end{tabular}

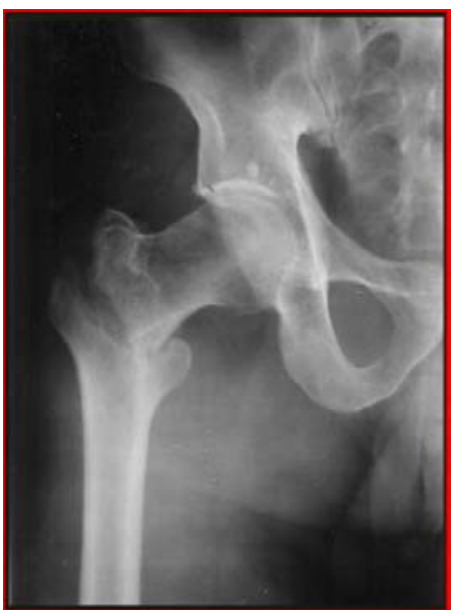

Fig 1: Preoperative Xray

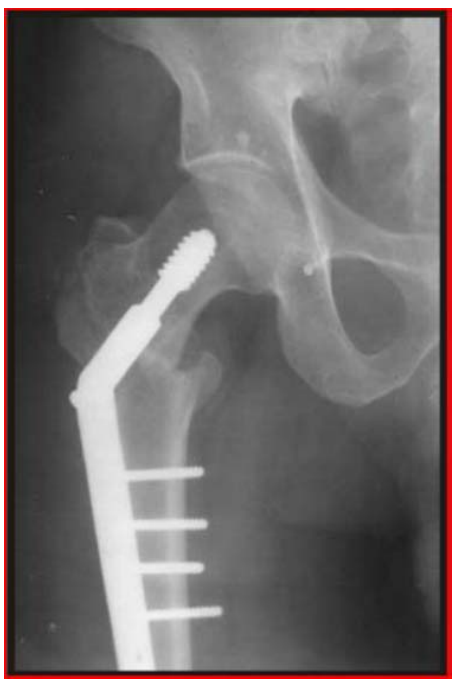

Fig 2: Immediate postop xray

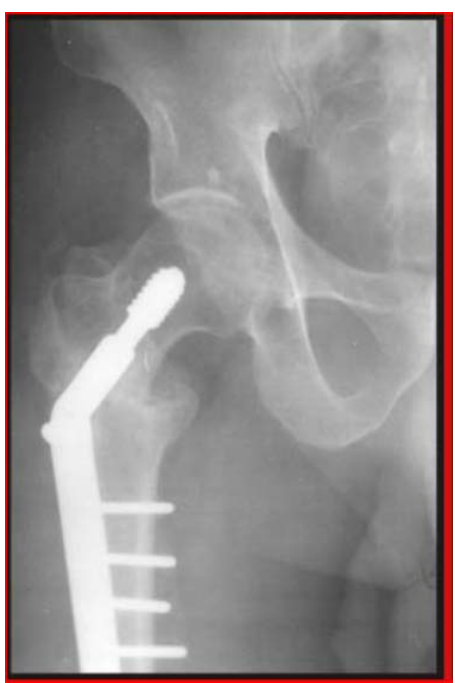

Fig 3: After 3 months

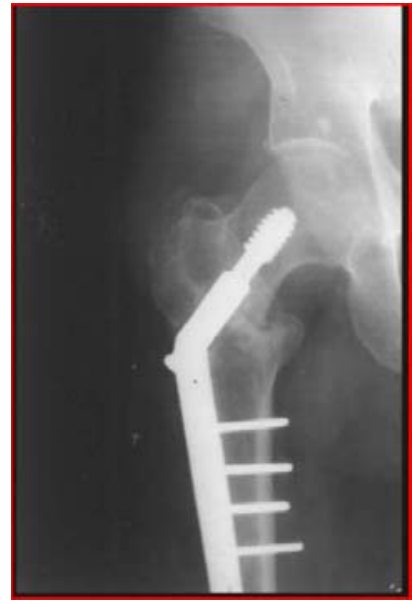

Fig 4: After 6 months

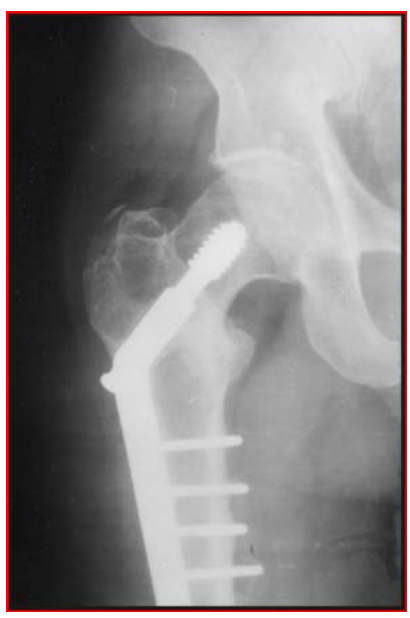

Fig 5: After one year

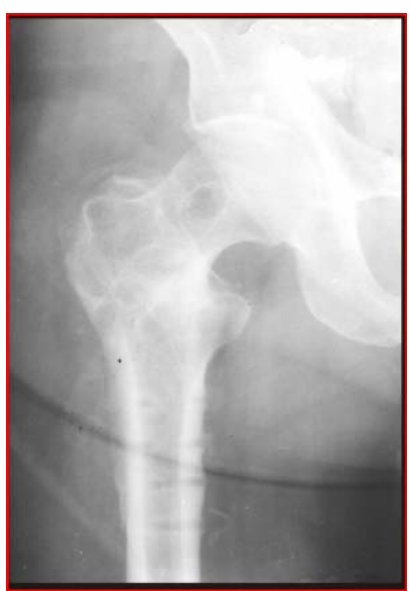

Fig 6: After implant removal

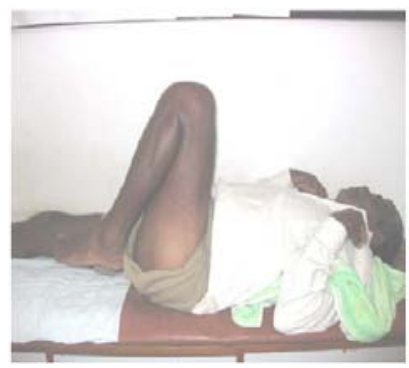

Fig 7: Active hip flexion 


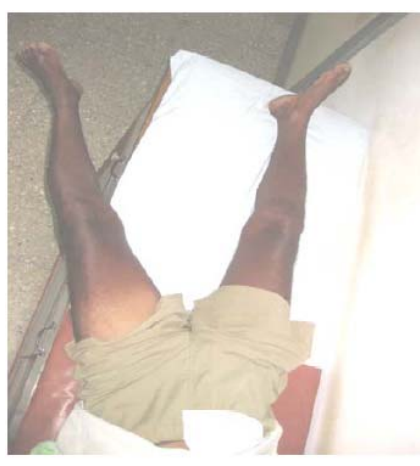

Fig 8: Active abduction

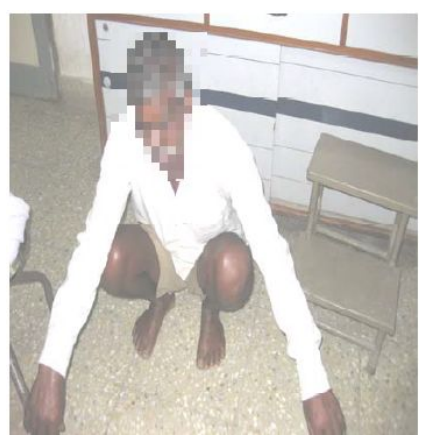

Fig 9: Squatting

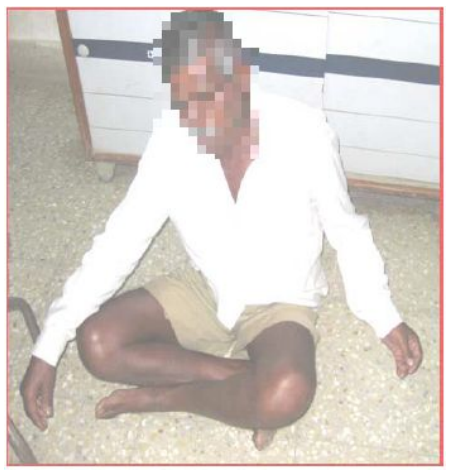

Fig 10: Sitting Crosslegged

\section{Discussion}

Intertrochanteric fractures of the femur are relatively common injuries among the elderly individuals. The Dynamic Hip Screw with Barrel Side Plate assembly is theoretically, practically and biomechanically more advantageous than other implants. It remains the best implant available for fixation of intertrochanteric fractures of the femur. In the present study, the average age for intertrochanteric fractures was 54.7 years ranging from 22 to 88 years which is comparable to the study by Arun Kumar ${ }^{[4]}$. Female to male ratio in this study is 30:70. The female preponderance in our study is not similar to the female preponderance observed by various other authors like JohnH. Doherty et al [5], Christopher IA etal ${ }^{[6]}$ and Brian ${ }^{[7]}$. This is because of the fact that the Indian males have higher risk of osteoporosis than the western counter parts ${ }^{[8]}$. A nutritional factor, including decreased calcium has been implicated as the cause. $80 \%$ of the cases in this series were due to a fall, such as at home, slipping in the bathroom or missing a step. In 6 patients the mode of injury was RTA. The higher incidence of intertrochanteric fractures in the elderly due to a trivial trauma is comparable to other studies done by Hornby et al ${ }^{[9]}$. Pain is an important criterion for the evaluation of intertrochanteric fractures. Following surgery pain in the hip joint may be due to mechanical complications like screw cutout, joint penetration or infection, or due to avascular necrosis of the femoral head or secondary osteoarthritis of the hip joint. In the present study, only three patients had pain in the hip joint at the end of six months. In one patient the pain was due to a mechanical complication, i.e. screw cutout. In the other two patients there was no mechanical complication, or infection or secondary changes, but the patient still complained of pain in the hip joint. In the Malcolm L. Ecker et al ${ }^{[10]}$ series, $79 \%$ showed no pain, $18 \%$ had mild pain and $1 \%$ had moderate pain. The ranges of movements were full range to fair in $96.3 \%$ of patients, which is comparable to Max P. Esser et al ${ }^{[11]}$ series. In the present study at the end of six months, 24 patients had no shortening, 6 patients had shortening varying from 1.5 to $2 \mathrm{~cm}$. In intertrochanteric fractures 0.5 to $1 \mathrm{~cm}$ of shortening is acceptable. In the series by Sernbo et al ${ }^{[12]}$ shortening was seen in $20 \%$ of the cases. In the present study eighteen patients (60\%) showed union by twelve weeks and eleven patients (33\%) showed union by sixteen weeks. The average time for union was 13.2 weeks. This is comparable to studies done by Mohanty and Chacko ${ }^{[13]}$ and Wolfgang et al ${ }^{[14]}$. In the present study there were no cases of implant failure, such as breakage of the plate, breakage of the screw or separation of the screw from the plate etc.

\section{Conclusion}

This study shows that intertrochanteric fracture is common in elderly population with male preponderance mainly occurring between 51-65 years. Common mode of injury being trivial fall, right side being slightly more involved in injury. Early operative intervention with Dynamic hip screw with $135^{\circ}$ side plate with barrel gives good results, helps early mobilization of elderly patients decreasing morbidity \& mortality and achieves rigid fixation even in osteoporotic bone.

\section{References}

1. Ray Marks. Hip fracture epidemiological trends, outcomes, and risk factors, 1970-2009. Int J Gen Med, 2010; 3:1-17.

2. Hedlund R, Lindgren U, Ahlbam A. Age and sex specific incidence of femoral neck and trochanteric fractures: an analysis based on 20,538 fractures in Stockholm county, Sweden, 1972-81. Clin Orthop Rel Res, 1987; 222:132.

3. Robert W Bucholz, James D Heckman, Charles M CourtBrown, Rockwood and Green's Fractures in Adults, 2(6):1827-1844.

4. Kumar AK, Thong G, Laloo N, Singh AM, Singh SN. Management of intertrochanteric fractures. IJ Orth, 2006; 40(2):100-102.

5. Doherty JH, Lyden JP. Intertrochanteric fractures of the Hip treated with the Hip Compression Screw. Clinical Orthopaedics and Related Research, 1979; 141:184-187.

6. Christopher IA, Robinson CM. Prospective Randomized Controlled Trial of an Intramedullary Nail Versus Dynamic Screw and Plate for Intertrochanteric Fractures of the Femur. Journal of Orthopaedic Trauma, 2001; 15(6):394-400.

7. Aros B, Anna NA, Tosteson, Daniel JG, Kenneth J Koval. Is a Sliding Hip Screw or IM Nail the Preferred Implant for Intertrochanteric Fracture Fixation. Clin Orthop Relat Res, 2008; 466:2827-2832. 
8. Nordin Bec. International patterns of osteoporosis. Clin Orthop, 1966, 17-30.

9. Hornby R et al. Operative or Conservative Treatment for Trochanteric Fractures of the Femur. Journal of Bone and Joint Surgery, 1989; 71B:619-623.

10. Ecker ML. The Treatment of Trochanteric Hip Fractures using a Compression Screw. Journal of Bone and Joint Surgery, 1975; 57A: 23-27.

11. Esser MP, Kassab JY, Jones DHA. Trochanteric Fractures of the Femur. Journal of Bone and Joint Surgery, 1986; 68B:557-560.

12. Sernbo I, Johnell O, Gentz CF et al. Unstable Intertrochanteric Fractures of the Hip. Journal of Bone and Joint Surgery, 1988; 70A:1297-1303.

13. Mohanty SP, Chacko V. A Comparative Analysis of Operative and Nonoperative Management of Trochanteric Fractures. Indian Journal of Orthopaedics 1984; 18(1).

14. Wolfgang GL. Treatment of Intertrochanteric Fracture of the Femur using Sliding Screw Plate Fixation. Clinical Orthopaedics and Related Research, 1982; 163:148-158. 